\title{
Electrical Resistance Characterization of Strain-Induced Multiwalled Carbon Nanotubes Using MEMS-Based Strain Engineering Device
}

\author{
Kenji Yamauchi, Keisuke Obata, Shinya Nakata, \\ Koji Sugano, Kisaragi Yashiro, ${ }^{1}$ and Yoshitada Isono* \\ Graduate School of Engineering, Kobe University, 1-1 Rokkodai, Nada, Kobe 657-8501, Japan \\ ${ }^{1}$ Graduate School of Engineering, Gifu University, 1-1 Yanagido, Gifu 501-1193, Japan
}

(Received August 28, 2015; accepted October 20, 2015)

Keywords: multiwalled carbon nanotubes, electrical resistance, strain, MEMS

In this research, we clarified the electrical resistance change and mechanical properties of straininduced multiwalled carbon nanotubes (MWCNTs), synthesized by atmospheric chemical vapor deposition (CVD), using a MEMS-based strain engineering device mounted on an in situ scanning electron microscopy (SEM) nanomanipulation system. The Young's modulus of an individual MWCNT and its shear strength during interlayer sliding deformation were estimated from the loaddisplacement curve. The electrical resistance of the MWCNT was $215 \mathrm{~kW}$ without strain, while an anomalous electrical resistance change was observed under a large strain. The resistance change ratio was almost 0 during the interlayer sliding of the MWCNT, but it specifically showed a sharp increase at the end of the sliding in spite of the MWCNT not breaking mechanically. Molecular dynamics (MD) simulations provided us with a reasonable suggestion that the hard sticking with an atomic reconfiguration at the edge of the outer layer of the MWCNT induces the sharp increase in electrical resistance before the mechanical breaking of the MWCNT.

\section{Introduction}

Multiwalled carbon nanotubes (MWCNTs) are one of the promising materials as ultralowresistance interconnects for the via structures of a half-pitch $32 \mathrm{~nm}$ fabrication node and beyond, ${ }^{(1-3)}$ because they cannot lead to the electromigration often observed in metallic interconnections. The high stiffness and low electrical resistance of MWCNTs are also suitable for interconnect materials. However, via structures in high-density integrated circuit boards suffer from the thermal strain generated in the device operation, which will lead to the damage or interlayer sliding deformation of MWCNTs, resulting in the change in electrical resistance. Thus, basic information on electrical resistivity for a mechanically strained individual MWCNT is required for a reliable design of MWCNT interconnects.

For characterizing the mechanical properties of one-dimensional nanostructure materials such as individual CNTs and carbon- and silicon-related nanowires, MEMS-based strain engineering devices have so far been developed. ${ }^{(4-10)}$ Most of these devices include electrostatically or thermally driven actuators to produce a tensile force and displacement sensing elements such as a differential ${ }^{*}$ Corresponding author: e-mail: isono@mech.kobe-u.ac.jp 
capacitive displacement sensor or a gauge structure. The authors have proposed a couple of devices including an electrostatically driven actuator and a capacitive displacement sensor incorporated into a cantilever motion amplification system, and have successfully clarified the mechanical properties of carbon nanowires, silicon nanowires and MWCNTs. ${ }^{(4-7)}$ In the mechanical characterization of individual MWCNTs synthesized by chemical vapor deposition (CVD), ${ }^{(11)}$ the interlayer sliding deformation accompanied by repeated stick-slip and hard sticking events was observed under uniaxial loading, and the shear strength during the sliding was directly derived from the shear interaction force. However, the electrical resistance has not been evaluated for strain-induced MWCNTs; thus, the reliability of MWCNT interconnects under operation cannot be ensured.

In the present research, we focus on performing a complete mechanoelectric characterization of an individual MWCNT under uniaxial tensile strain, and also report on molecular dynamics (MD) tensile simulations of telescopic MWCNT models using the bond-order potential function for understanding the electrical resistance change during interlayer sliding deformation on an atomic scale.

\section{Experimental and Analytical Methodologies}

\subsection{MEMS-based strain engineering device}

The design and dimensions of the MEMS-based strain engineering device are basically the same as those of the previously developed devices. ${ }^{(4-6)}$ Figure 1 shows a schematic of the strain engineering device and its lumped mechanical model. An MWCNT is individually bridged at

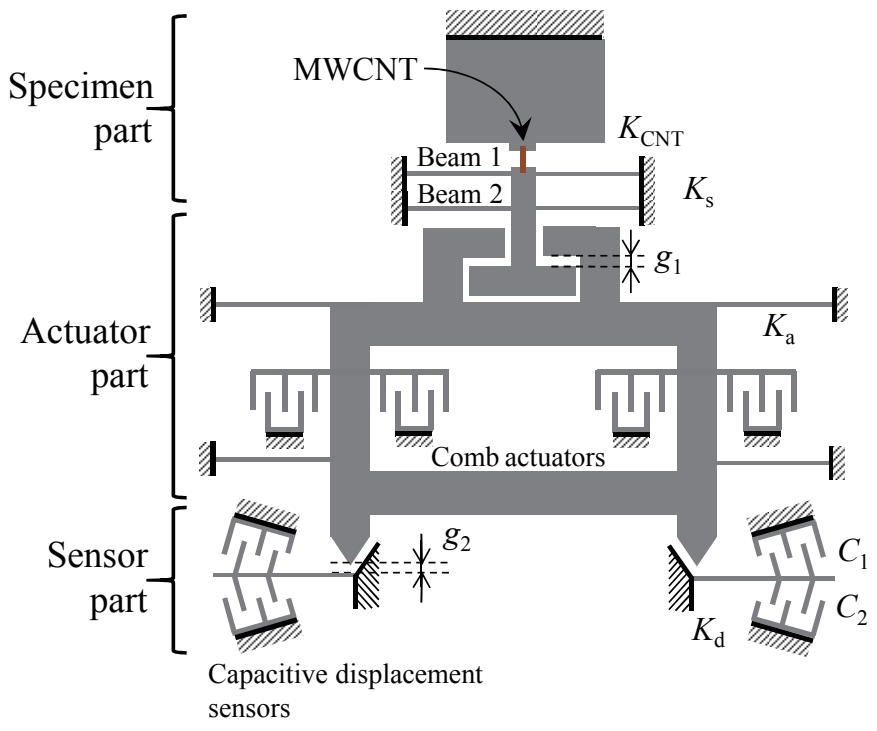

(a)

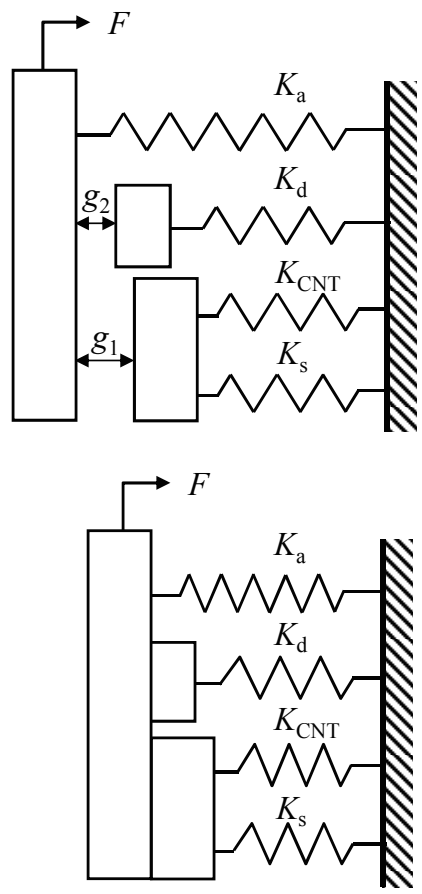

(b)

Fig. 1. Schematics of (a) MEMS-based strain engineering device and (b) its lumped mechanical model. 
the $5 \mu \mathrm{m}$ gap in the specimen part in Fig. 1 using the in situ scanning electron microscopy (SEM) nanomanipulation system. An electrostatically driven comb actuator is separately arranged at an interval of $4 \mu \mathrm{m}\left(=g_{1}\right)$ from the movable portion of the specimen part. Capacitive displacement sensors are set at an interval of $3 \mu \mathrm{m}\left(=g_{2}\right)$ from the lower part of the comb actuator employed for the tensile loading system. The movable portion of the specimen part and the comb actuator are suspended by each doubly clamped supporting beam from fixed anchor parts. The capacitive displacement sensors are supported by cantilever beams.

As shown in the lumped mechanical model, the force generated by the comb actuator deforms the supporting beams of the comb actuator with a stiffness $K_{\mathrm{a}}$ at the beginning of device operation. The force is continually spent for the elastic deformation of the capacitive displacement sensor with a stiffness $K_{\mathrm{d}}$, the supporting beams of the specimen area with a stiffness of $K_{\mathrm{s}}$, and an individual MWCNT. Thus, $F$ is expressed as

$$
F=K_{\mathrm{a}} g_{2}+\left(K_{\mathrm{a}}+K_{\mathrm{d}}\right)\left(g_{1}-g_{2}\right)+\left(K_{\mathrm{a}}+K_{\mathrm{d}}+K_{\mathrm{s}}\right)\left[\delta_{\mathrm{a}}^{\text {before }}-g_{2}\right]+P .
$$

Here, $\delta_{\mathrm{a}}^{\text {before }}$ is the displacement produced by the comb actuator before the failure of the MWCNT, and $P$ is the tensile force of the sample. Because $F$ before the failure of the MWCNT is equal to that after its failure at each voltage applied to the comb actuator, $P$ is described as

$$
P=\left(K_{\mathrm{a}}+K_{\mathrm{d}}+K_{\mathrm{s}}\right)\left(\delta_{\mathrm{a}}^{\text {after }}-\delta_{\mathrm{a}}^{\text {before }}\right) .
$$

Here, $\delta_{\mathrm{a}}^{\text {after }}$ is the actuator displacement of the device without the sample after its failure. The stiffnesses $K_{\mathrm{a}}$ and $K_{\mathrm{s}}$ display the nonlinearity due to the tensile stress in the longitudinal direction of the doubly clamped supporting beams under a large deformation. ${ }^{(12)}$ Thus, the stiffnesses $K_{\mathrm{a}}$ and $K_{\mathrm{s}}$ were calculated by the following equation derived from the principle of minimum potential energy:

$$
K=\left(\frac{\pi^{4}}{384}\right)\left\{\frac{E h\left(a^{3}+a^{2} b+a b^{2}+b^{3}\right)}{l^{3}}\right\}+\left(\frac{\pi^{4}}{256}\right)\left\{\frac{E h(a+b)}{l^{3}}\right\} \delta_{\mathrm{a}}^{2},
$$

where $E$ is the Young's modulus of the beam, $l$ is the beam length, $\delta_{\mathrm{a}}$ is the actuator displacement, $a$ and $b$ are the upper and lower widths of the beam, respectively, and $h$ is the beam height. The stiffness calculated using Eq. (3) agrees with that obtained by finite element analyses, although it is omitted here.

The differential capacitors with arc-shaped combs incorporated into the cantilever motion amplification system were also integrated into the device as the capacitive displacement sensors. They were designed to achieve a scale factor larger than $1 \mathrm{fF} / \mathrm{nm}$, which carried an advantage of a large capacitance change for an ultrasmall displacement and an accurate calibration of the sensor. The sensor design was described in detail in Ref. 6. The charge amplifier circuit board manufactured by the authors was connected to the common electrode of the differential capacitors with arc-shaped combs. Figure 2 shows a schematic block diagram of the charge amplifier circuit. Modulation pulses of $\pm 5 \mathrm{~V}$ were applied at $1 \mathrm{MHz}$ from the synchronous modulator to the fixed electrodes with the arc-shaped comb structure opposite to the movable cantilevers. Manual adjustors for the offset voltage, gain, and phase were also installed in the amplifier circuit, and they were regulated before the experiment. 


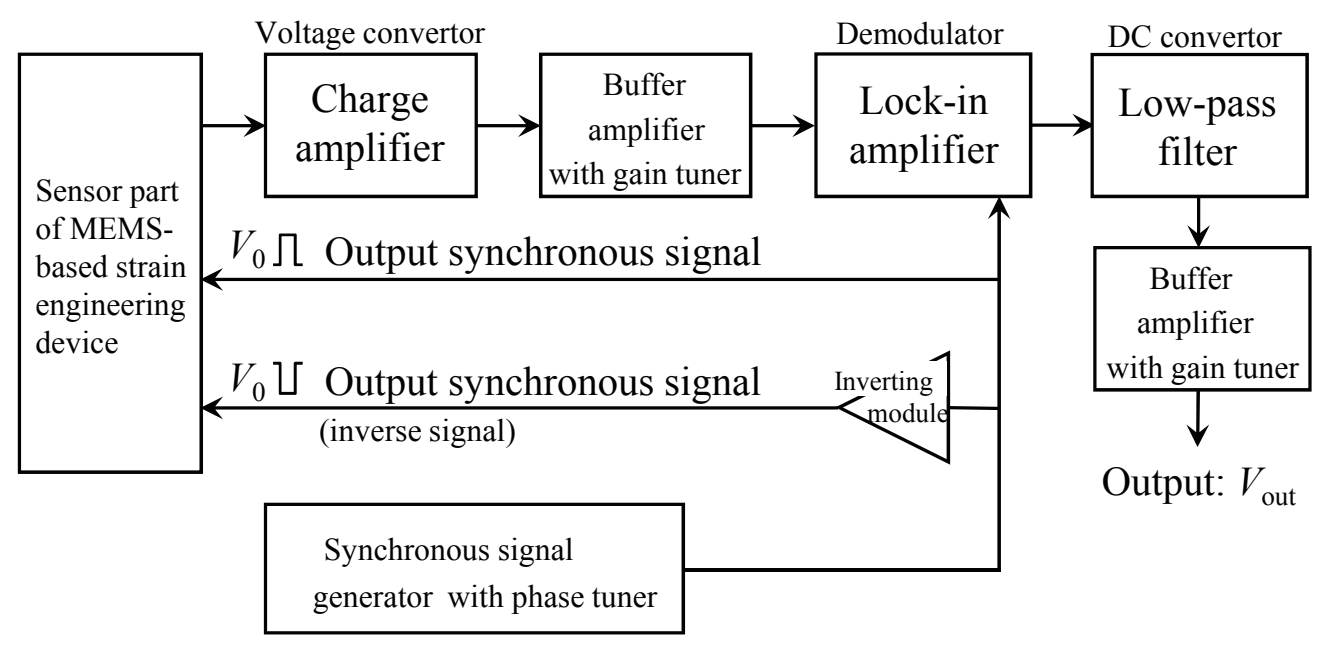

Fig. 2. Block diagram of the charge amplifier circuit connected to the capacitive displacement sensor.

\subsection{Fabrication}

Figures 3(a)-3(f) show the fabrication process for the strain engineering device. The starting substrate was a silicon ( $\mathrm{Si}$ )-on-insulator wafer with a $35-\mu \mathrm{m}$-thick single-crystal $\mathrm{Si}$ device layer and a 3- $\mu \mathrm{m}$-thick buried oxide layer on a $400-\mu \mathrm{m}$-thick single-crystal Si substrate, which was covered by a 400 -nm-thick $\mathrm{SiO}_{2}$ layer on the top and back surfaces [see Fig. 3(a)]. First, the areas of the electrostatically driven comb actuator and capacitive displacement sensor were doped by a diffusion process using boron as the dopant [see Fig. 3(b)]. The device structure was patterned on the device layer side after chromium film deposition. The back-surface patterning was also performed in a similar manner [see Fig. 3(c)]. The substrate layer was etched by deep-reactive ion etching (D-RIE), followed by the etching of the device layer [see Fig. 3(d)]. A vaporized hydrofluoric solution removed the buried oxide layer to release the movable parts of the device, and gold electrodes were deposited on the device by a stencil mask technique [see Fig. 3(e)]. The device was finally set on the printed circuit board with wire bonding between the board and the device, and an MWCNT was fixed at the specimen part by the in situ SEM nanomanipulation system [see Fig. 3(f)].

Figures 4(a)-4(c) show the SEM images of the fabricated MEMS-based strain engineering device. Each functional element was fabricated well on the device according to the design dimensions. Table 1 gives the design and measured values of the dimensions and stiffness for each beam used in the strain engineering devices after fabrication. The cross-sectional geometries of the beams were measured 10 times from the top and back surfaces. ${ }^{(4)}$ The length and diameter of the MWCNT tested in this work are also listed in the table. 


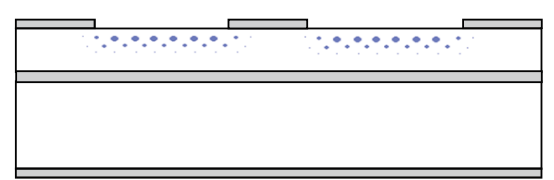

(a)

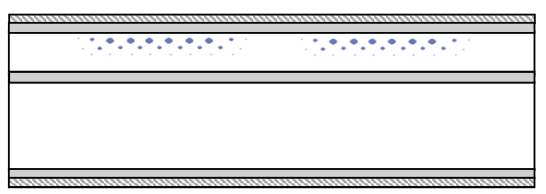

(b)

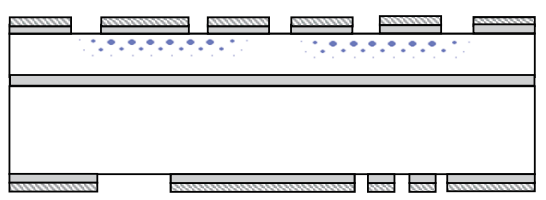

(c)

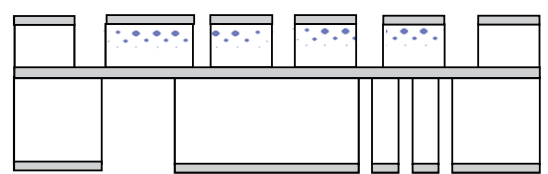

(e)

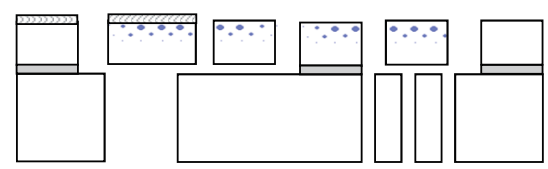

(d)

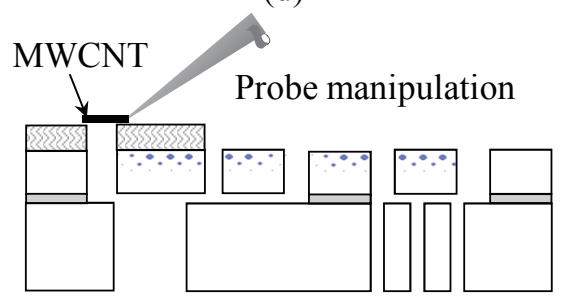

(f)

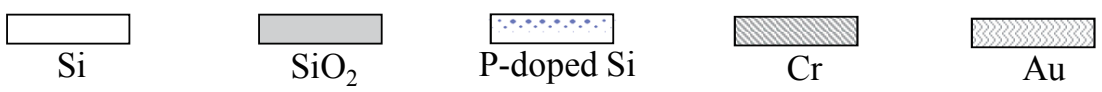

Fig. 3. Fabrication process flow of the MEMS-based strain engineering device.
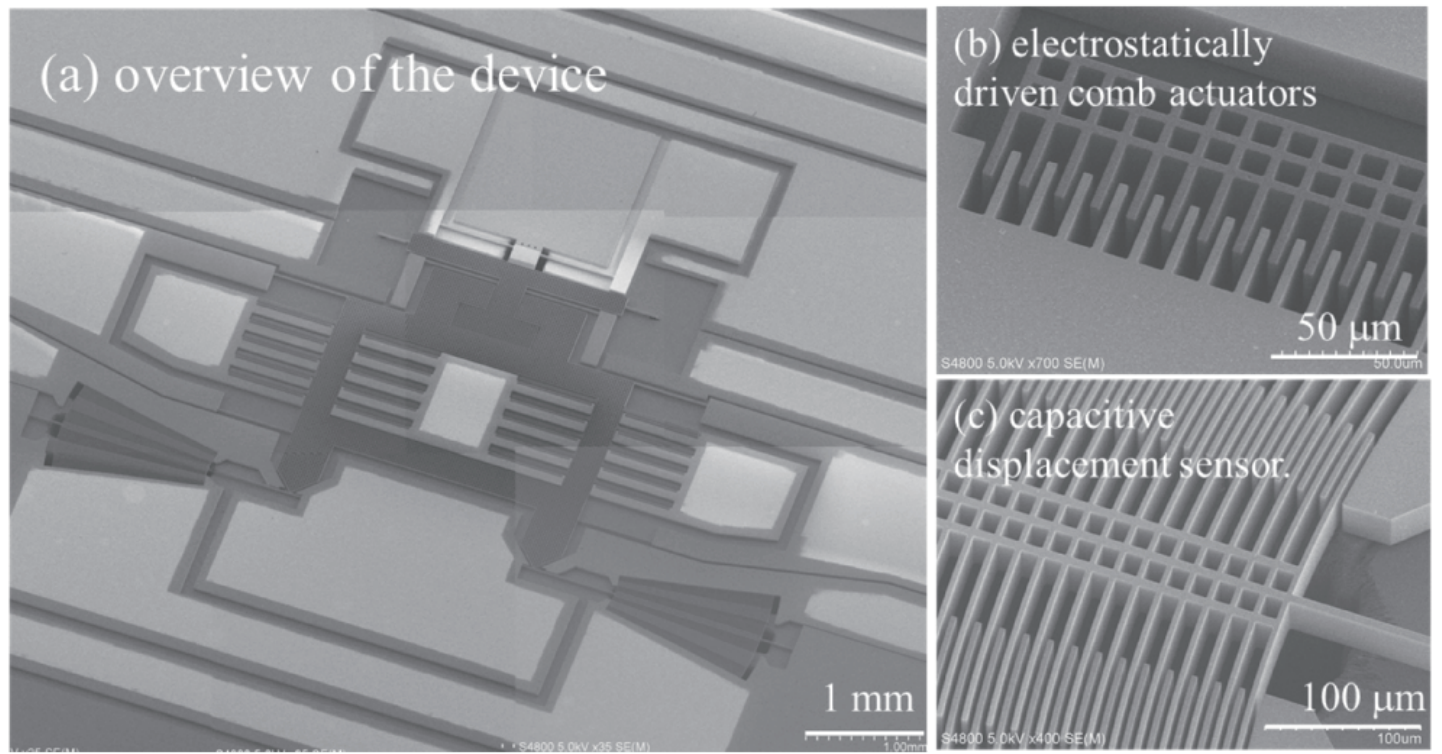

Fig. 4. SEM images of the MEMS-based strain engineering device: (a) overview of the device, (b) electrostatically driven comb actuators, and (c) arc-shaped capacitive displacement sensor. 
Table 1

Nominal dimensions of supporting beams and cantilevers used in the MEMS-based strain engineering device, and geometry of the sample.

\begin{tabular}{|c|c|c|c|}
\hline \multirow{2}{*}{ Beams } & Dimensions (unit) & Design & Measured (average) \\
\hline & Height $(\mu \mathrm{m})$ & 35 & 35 \\
\hline \multirow{5}{*}{$\begin{array}{l}\text { Supporting beams of } \\
\text { comb drive actuator }\end{array}$} & Length $(\mu \mathrm{m})$ & 700 & 697.1 \\
\hline & Upper width $(\mu \mathrm{m})$ & 5 & 4.215 \\
\hline & Lower width $(\mu \mathrm{m})$ & - & 3.578 \\
\hline & Number of beams & 4 & $\leftarrow$ \\
\hline & Total stiffness, $K_{\mathrm{a}}(\mathrm{N} / \mathrm{m})$ & Eq. (3) & $\leftarrow$ \\
\hline \multirow{6}{*}{$\begin{array}{l}\text { Cantilever of capacitive } \\
\text { displacement sensor }\end{array}$} & Length, $l_{2}(\mu \mathrm{m})$ & 110 & 110 \\
\hline & Upper width $(\mu \mathrm{m})$ & 3.4 & 2.643 \\
\hline & Lower width $(\mu \mathrm{m})$ & - & 1.683 \\
\hline & Number of cantilevers & 2 & $\leftarrow$ \\
\hline & Total stiffness, $K_{\mathrm{d}}(\mathrm{N} / \mathrm{m})$ & 87.33 & 23.59 \\
\hline & Scale factor $(\mathrm{fF} / \mathrm{nm})$ & 1.26 & - \\
\hline \multirow{5}{*}{$\begin{array}{l}\text { Supporting beam } 1 \\
\text { at specimen part }\end{array}$} & Number of beams at one side & 2 & $\leftarrow$ \\
\hline & Length, $L_{1}(\mu \mathrm{m})$ & 700 & 695.8 \\
\hline & Upper width $(\mu \mathrm{m})$ & 7 & 6.174 \\
\hline & Lower width $(\mu \mathrm{m})$ & - & 5.518 \\
\hline & Total stiffness $(\mathrm{N} / \mathrm{m})$ & Eq. (3) & $\leftarrow$ \\
\hline \multirow{5}{*}{$\begin{array}{l}\text { Supporting beam } 2 \\
\text { at specimen part }\end{array}$} & Number of beams at one side & 2 & $\leftarrow$ \\
\hline & Length, $L_{2}(\mu \mathrm{m})$ & 700 & 696.4 \\
\hline & Upper width $(\mu \mathrm{m})$ & 5 & 4.158 \\
\hline & Lower width $(\mu \mathrm{m})$ & - & 3.578 \\
\hline & Total stiffness $(\mathrm{N} / \mathrm{m})$ & Eq. (3) & $\leftarrow$ \\
\hline \multirow{2}{*}{ Sample } & Length $(\mu \mathrm{m})$ & & 8.55 \\
\hline & Diameter (nm) & & 36.4 \\
\hline
\end{tabular}

\section{Device Characterizations}

\subsection{Electrostatically driven comb actuator}

For the calibration of the sensor, the displacement generated by the comb actuator was precisely measured as the position at the free end of the capacitive displacement sensor using a chargecoupled device (CCD) camera system mounted on an optical microscope, ${ }^{(4)}$ since the deflection of the cantilever set into the sensor was amplified by as much as 32.5 times against the actuator displacement. Figure 5 shows the variation in displacement at the free end of the sensor with an increase in applied voltage to the actuator. The displacement was measured in air because the previous work confirmed that there was no clear distinction between the measured data in air and in vacuum. ${ }^{(6)}$ No displacement of the sensor is observed below $20 \mathrm{~V}$ attributed to the gap set between the actuator and sensor parts. The voltages when the cantilever displacement begins to increase are larger than that calculated using the measured dimensions of the device structure. This is attributed to the widening of the gap between the comb actuator and the sensor in the D-RIE process. However, the increasing rate of displacement calculated by the measured dimensions to the voltage is consistent with the measured data. If the calculation curve is simply shifted to the measurements 


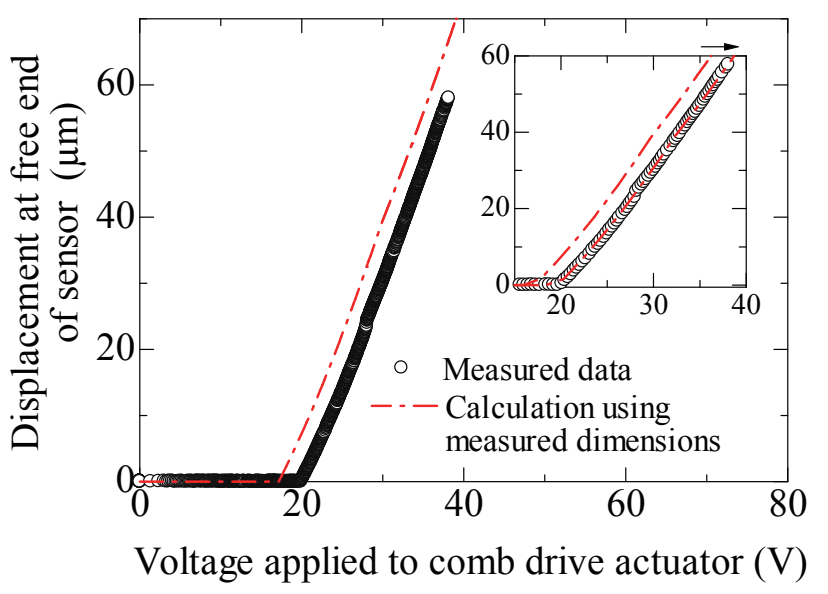

Fig. 5. (Color online) Variation in displacement at the free end of the sensor with increasing voltage applied to the comb drive actuator.

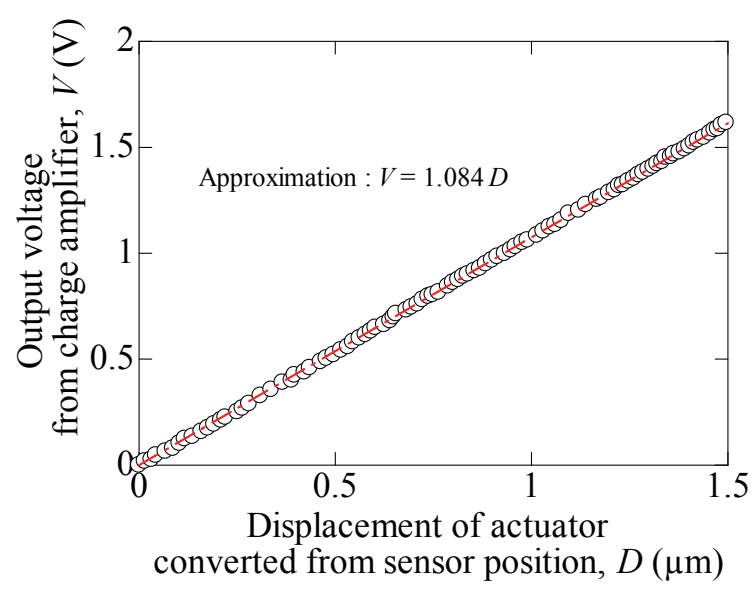

Fig. 6. (Color online) Relationship between the output voltage from the charge amplifier circuit and the displacement of the comb drive actuator.

as shown in the inset in Fig. 5, the maximum error of the calculation from the experiment is $3.2 \%$ at $38 \mathrm{~V}$. Thus, the stiffness based on the measured dimensions of the supporting beams was estimated well.

\subsection{Capacitive displacement sensor}

Figure 6 shows the sensitivity of the charge amplifier circuit connected to the capacitive displacement sensor. The displacement of the actuator is converted from the position at the free end of the sensor. The linear relationship between the output voltage and the actuator displacement is recognized as a function of $V=1.084 D$, which suggests that the fabrication error in the gap distance between the comb actuator and the sensor on both sides can be neglected. The output signals were recorded in a memory with a 14 bit $\mathrm{AD}$ converter working in the range of $\pm 5 \mathrm{~V}$ at a period of 10 $\mathrm{ms}$. Consequently, the measurement resolution of the displacement was $0.501 \mathrm{~nm}$ in this case. The measurement resolution of the force was $47.5 \mathrm{nN}$, which was derived from the total stiffness of the supporting beams calibrated by the previously reported method. ${ }^{(4)}$

\section{MD Simulations}

To understand the deformation behavior after the interlayer sliding of MWCNTs under uniaxial tensile strain, MD tensile simulations are carried out using three MWCNT models with cylindrical carbon layers alternatively placed like a telescope, as shown in Fig. 7. In the model composed of two cylindrical layers, both ends of the outer layers are fixed and the inner layer is placed at the center of the model. In the models with three and five cylindrical layers, both ends of the oddnumbered layers from the inside of the model are fixed; the even-numbered layers are positioned at the center of each model. The carbon cylinder has a zigzag structure in the longitudinal direction. The bond length $b$ is $0.145 \mathrm{~nm}$ and 4 green atoms in the figure are the minimum number of units of the atomic configuration in the circumferential direction of the carbon ring. The minimum core of 

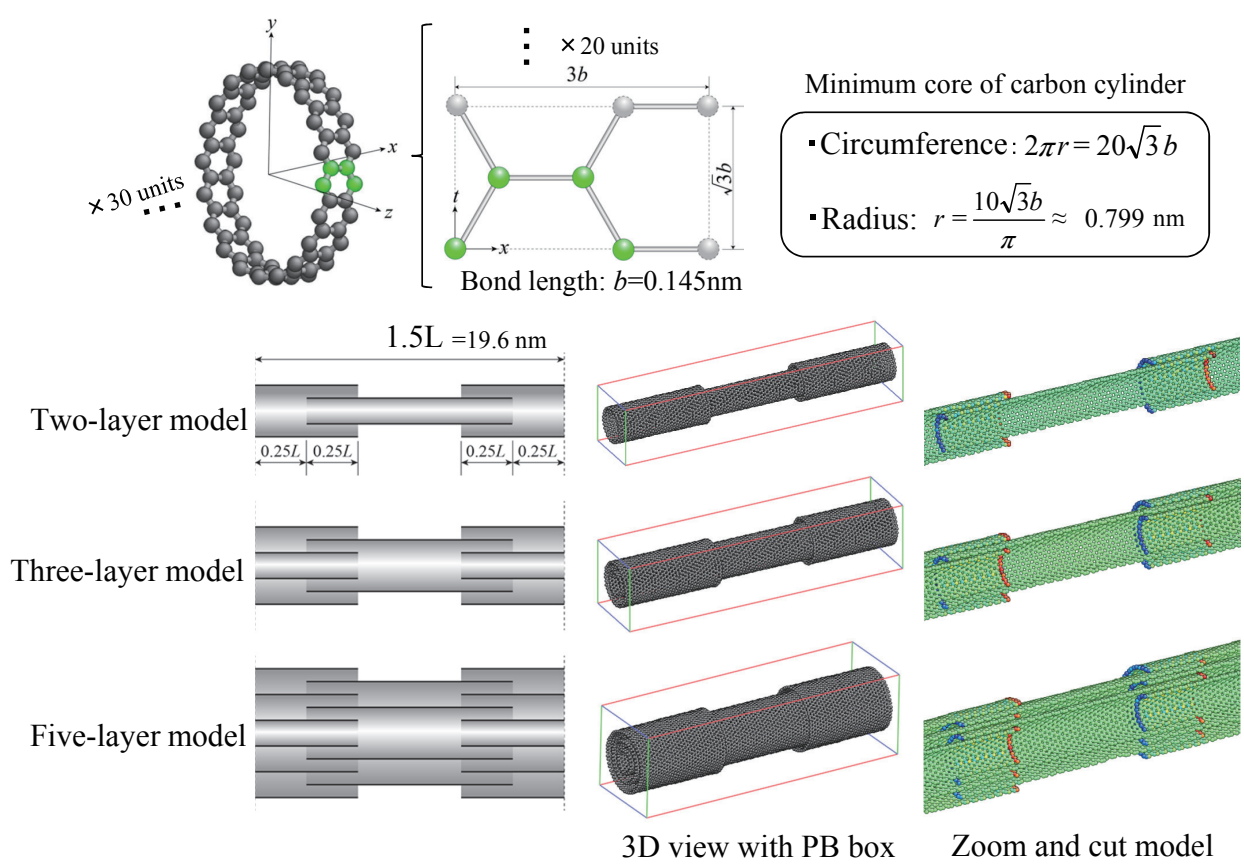

Fig. 7. (Color online) Schematics of the MWCNT models with alternatively placed cylindrical carbon layers for the MD tensile simulations.

the carbon cylinder is composed of 20 units in the circumference, which results in a circumference of $5.023 \mathrm{~nm}$ and a radius of $0.799 \mathrm{~nm}$. The interval distance between layers is assumed to be 0.35 $\mathrm{nm}$, so that the 2nd, 3rd, 4th, and 5th layers of the MWCNT model counted from the minimum core consist of 29, 38, 47, and 56 units in the circumferential direction, respectively. The cylindrical layers placed at the center of the models are composed of 30 unit rings in the longitudinal direction, and their length is $13.05 \mathrm{~nm}(=L)$. Thus, the total length of the telescopic MWCNT models is 19.6 $\mathrm{nm}(=1.5 \mathrm{~L})$, which corresponds to the periodic boundary length, since the overlap region between the outer and inner layers is defined as $0.25 \mathrm{~L}$ as shown in Fig. 7.

The classical MD-simulated tensile testing of the telescopic MWCNT models using the bondorder empirical potential function was proposed by Brenner. ${ }^{(13)}$ The bond-order potential used is expressed as

$$
E=\sum_{i} \sum_{j>i}\left[V_{\mathrm{R}}\left(r_{i j}\right)-\overline{B_{i j}} V_{\mathrm{A}}\left(r_{i j}\right)\right]
$$

Here, $E$ is the binding energy for the carbon potential, which is the sum of the repulsive and attractive terms given by

$$
V_{\mathrm{R}}\left(r_{i j}\right)=f_{\mathrm{c}}\left(r_{i j}\right) \frac{D_{i j}^{(\mathrm{e})}}{S_{i j}-1} \exp \left[\sqrt{2 S_{i j}} \beta_{i j}\left(R_{i j}^{(\mathrm{e})}-r\right)\right],
$$

and 


$$
V_{\mathrm{A}}\left(r_{i j}\right)=f_{\mathrm{c}}\left(r_{i j}\right) \frac{S_{i j} D_{i j}^{(\mathrm{e})}}{S_{i j}-1} \exp \left[\sqrt{\frac{2}{S_{i j}}} \beta_{i j}\left(R_{i j}^{(\mathrm{e})}-r\right)\right],
$$

respectively. $f_{\mathrm{c}}\left(r_{\mathrm{ij}}\right)$ is the function that restricts the pair potential to nearest neighbors, and $\overline{B_{\mathrm{ij}}}$ is the empirical bond-order function. ${ }^{(13)}$ Other parameters in Eqs. (4)-(6) are indicated in Ref. 13. The Van der Waals force between carbon cylinders was assumed to be given by the Lennard-Jones potential function on the basis of the elastic constants of graphite, as follows:(14)

$$
U(r)=4 \varepsilon\left[\left(\frac{\sigma}{r}\right)^{12}-\left(\frac{\sigma}{r}\right)^{6}\right]
$$

Here, $U(r)$ is the potential energy between two atoms, $\sigma$ is the finite distance at which the interparticle potential is 0 , and $\varepsilon$ is the depth of the potential. $\sigma$ and $\varepsilon$ were assumed to be 0.3345 $\mathrm{nm}$ and $4.77 \times 10^{-3} \mathrm{eV}$, respectively, from Ref. 14 .

The periodic boundary condition was employed at the fixed ends of telescopic MWCNT models in the $x$-direction. To apply tensile strain to the models, the periodic boundary length was increased at $10^{-6}$ strain $(=\varepsilon)$ per time step after stabilizing the thermal energies of atoms. MD simulations were run at $0.1 \mathrm{~K}$ in the microcanonical ensemble. The time series of the position for atoms was calculated using the Newton's equation of motion using the Verlet algorithm at a time step of 0.1 fs.

\section{Results and Discussion}

\subsection{Tensile test of individual MWCNT}

The in situ SEM nanomanipulation system was employed to pick an individual MWCNT from a substrate and to fix it to the strain engineering device. The details of the nanomanipulation system are provided in Ref. 6. The MWCNT used in this work had an average external diameter of 36.4 $\mathrm{nm}$, and the distance between fixed points on the device was $8.55 \mu \mathrm{m}$.

Figure 8 shows the relationship between the tensile displacement converted from the output voltage of the capacitive displacement sensor and the voltage applied to the comb actuator. The

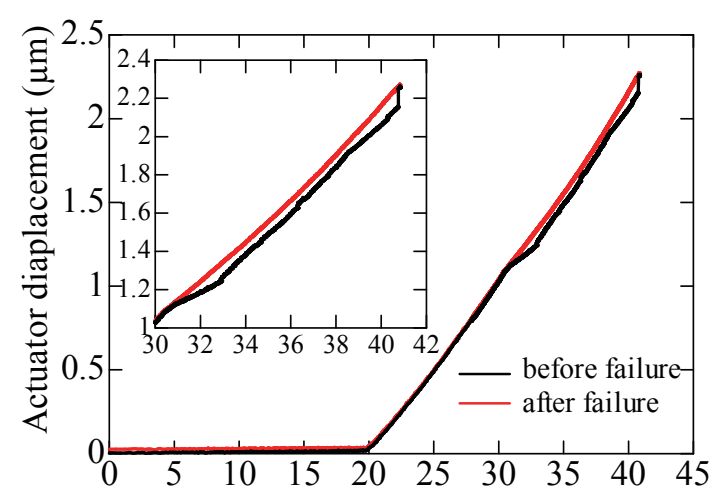

Voltage applied to comb drive actuator (V)

Fig. 8. (Coolor online) Variation in tensile displacement with increasing bias voltage applied to the comb drive actuator before and after failure of the sample. 
displacement increases above a voltage of $19.8 \mathrm{~V}$ because of the gap between the actuator and the sensor. The MWCNT was tensioned in the voltage range from 30.8 to $40.7 \mathrm{~V}$ since obtaining a clear difference in displacement before and after the failure of the MWCNT. The tensile load was estimated from this difference and the total stiffness of the supporting beams as shown in Eq. (2).

Figure 9 shows the tensile load-displacement curve of the MWCNT with the previously reported data depicted by solid lines. ${ }^{(6)}$ A linear increase in tensile load attributed to the elastic deformation is observed at the initial loading stage. However, the tensile load slightly decreases at a displacement of $0.1 \mu \mathrm{m}$, and it fluctuates in the range of 2 to $3 \mu \mathrm{N}$. This trend is similar to the previous work; ${ }^{(6)}$ the fluctuation region was formed by the interlayer sliding deformation with stickslip events of the individual MWCNT. The tensile load increases again above a displacement of 0.8 $\mu \mathrm{m}$ up to its sudden drop owing to the breaking of the sample. Young's modulus was determined to be $418 \mathrm{GPa}$ from the initial linear increase in load, which is close to the previously reported values by the authors, ${ }^{\left({ }^{6}\right)}$ but lower than those in other experiments. ${ }^{(15,16)}$ The lower modulus would have been affected by various topological defects in the MWCNTs such as vacancies, Stone-Wales defects and adatoms, which are inevitably introduced during their synthesis. ${ }^{(17,18)}$

\subsection{Electrical resistance change of ultrastrained individual MWCNT}

Figure 10 shows the electrical resistance change ratio during the tensile loading of the MWCNT along with its load-displacement curve. The electrical resistance of the MWCNT was $215 \mathrm{k} \Omega$ without strain. The resistance change ratio, $\left(R-R_{0}\right) / R_{0}$, was almost constant close to 0 during the elastic and interlayer sliding deformations. However, the sharp increase in the ratio was observed above a displacement of $0.8 \mu \mathrm{m}$, which is equivalent to $0.093 \varepsilon$ at the end of the sliding; that is, the MWCNT has electrically broken down in spite of it not breaking mechanically in the region of the second linear increase in load. The cause of the electrical breaking before the mechanical breaking will be discussed in the following section.

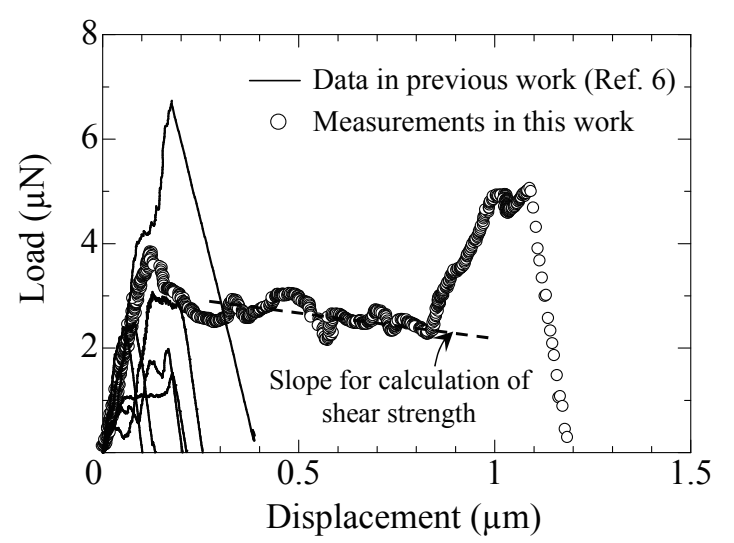

Fig. 9. Tensile load-displacement curves of the individual MWCNT with the previously reported data $^{(6)}$ depicted by solid lines.

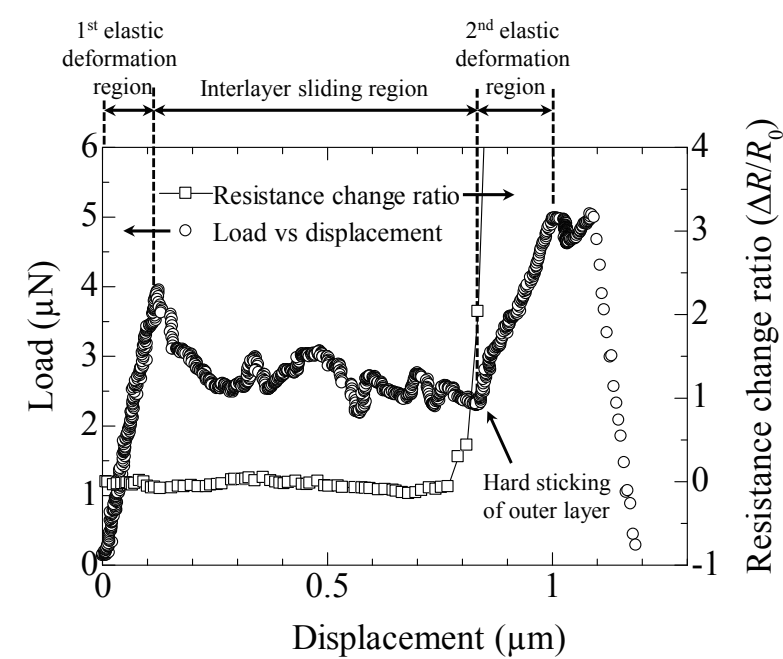

Fig. 10. Variation in electrical resistance change ratio with increasing tensile displacement. 
For a discussion of the anomalous electrical resistance change, the broken MWCNT was observed by SEM as shown in Figs. 11(a)-11(d). The fractured edge of the outer layer maintained a straight external form without plastic deformation, while the opposite edge showed a tiered external form produced by pulling the inside of the MWCNT. The fracture form of the MWCNT resulted from the sliding between interlayers like a telescopic motion. Using the diameter of the inner core extracted from the MWCNT, the interlayer shear strength was roughly estimated to be around 14.5 MPa using a single-shot extraction model. ${ }^{(19)}$ The shear strength was significantly larger than those obtained in other experiments for MWCNTs; ${ }^{(19,20)}$ the larger shear strength might be caused by the hard sticking between layers with stick-slip events. However, the cause of the sudden electrical resistance increase before the failure of the sample was not determined from the SEM observations.

\subsection{Interlayer sliding in MD simulations}

Figure 12 shows the relationship between the tensile reaction force and the displacement obtained in the tensile MD simulations. Pulling the two-layer model produces small force fluctuations of $\pm 1 \mu \mathrm{N}$ during the interlayer sliding, which is a telltale sign of stick-slip events between layers. The force also becomes 0 after pulling out the outer layer completely. A similar trend of the tensile force with an increase in displacement is observed for the three- and fivelayer models owing to sliding between interlayers, but the force in both models increases above a distance of $3.2 \mathrm{~nm}$ that corresponds to the overlap length of multilayers. In particular, the increased
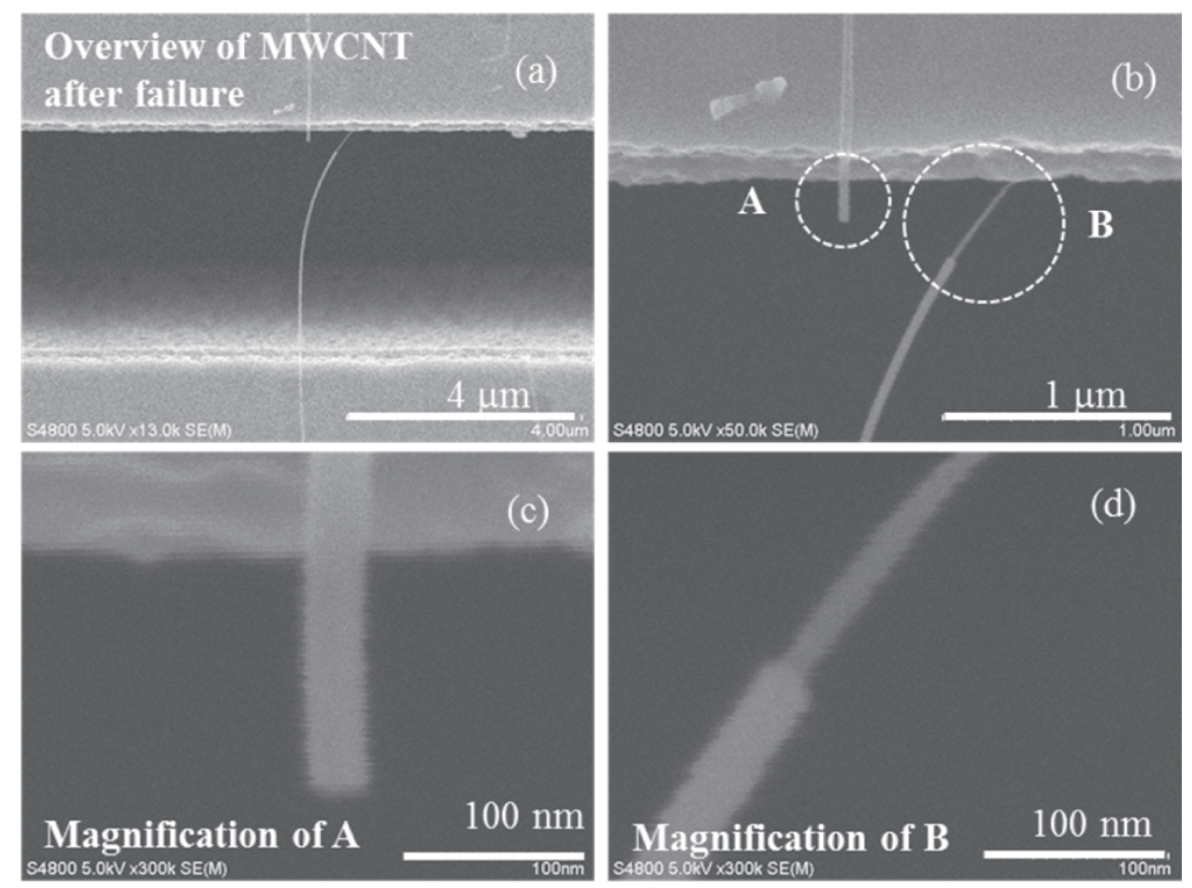

Fig. 11. SEM images of the sample after failure: (a) overview of the fractured sample, (b) magnified image of the breaking point, (c) magnified image of the breaking point for the outer layer, and (d) magnified image of the breaking point for the extracted inner layer. 


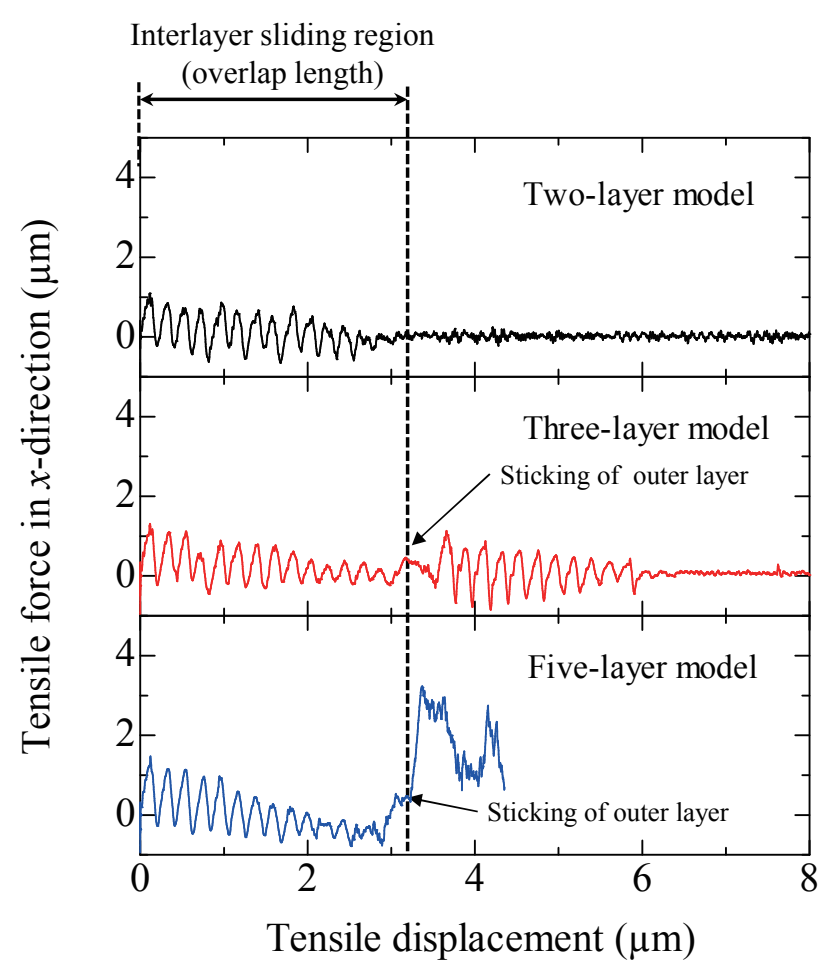

Fig. 12. (Color online) Tensile force-displacement curves calculated by MD simulations.

force in the five-layer model is maintained at a large value for a little while, and it decreases with an increase in displacement. The MD results of the tensile force after the interlayer sliding region qualitatively agree with the experimental result.

The snapshots of MWCNT models at the end of the interlayer sliding deformation are depicted in Figs. 13(a)-13(c). The interval distance between the inner and outer layers remains almost constant in the two-layer model, which maintains the tensile force in Fig. 12. However, in the three- and five-layer models, the edge atoms of the outer layer are strongly stuck to those of the inner layer, and the reconfiguration of the edge atoms is also recognized at the end of the sliding deformation. In the MD simulations, the increase in tensile force after the interlayer sliding was induced by the hard sticking of the edge atoms of the outer layer to the inner layer.

Other experiments have also revealed that interaction force of dangling bonds on the fractured edge of the outer layer to the inner layer, acting as an "edge effect", can induce hard sticking between layers. ${ }^{(19,20)}$ The sticking between layers can make the MWCNT mechanical strong, but may also make it electrically unstable owing to a reconfiguration of atoms at the interface between the layers; thus, the resistance change of the strain-induced MWCNT without mechanical breaking would have been produced. However, the essence of the anomalous resistance change for the strain-induced MWCNT cannot be concluded until further mechanoelectric characterization with a direct observation using a transmission electron microscope is performed. 


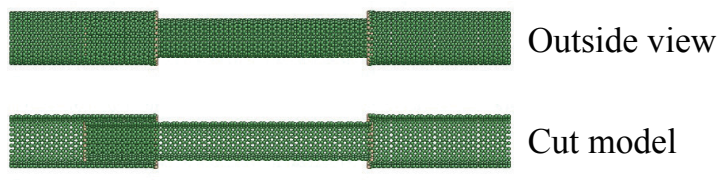

(a)

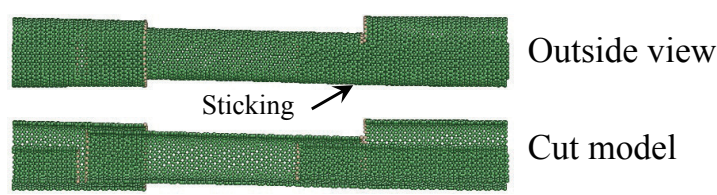

(b)

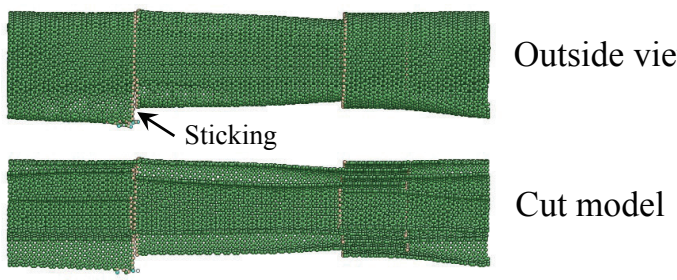

(c)

Fig. 13. (Color online) Snapshot images of the MWCNT models at the end of interlayer sliding deformation: (a) two-, (b) three-, and (c) five-layer models.

\section{Conclusions}

A MEMS-based strain engineering device was developed to clarify the electrical resistance change of a strain-induced MWCNT synthesized by CVD. The tensile test for an individual MWCNT was successfully carried out using the device mounted on an in situ SEM nanomanipulation system. The Young's modulus of the individual MWCNT was $418 \mathrm{GPa}$, and its interlayer shear strength was estimated to be $14.5 \mathrm{MPa}$ from a single-shot extraction model. MD tensile simulations of telescopic MWCNT models showed the stick-slip event in the tensile forcedisplacement relations, followed by an abrupt increase in tensile force. This trend was the same as the experiment qualitatively. The MD simulations suggested that the sharp increase in tensile force after the interlayer sliding was caused by the edge effect of the outer layer of the MWCNT, indicating the means of sticking the edge of the outer layer to the inner layer. The anomalous resistance change of the strain-induced MWCNT before mechanical breaking would have been produced by the edge effect attributed to the fractured edge of the outer layer.

\section{References}

1 F. Kreupl, A. P. Graham, G. S. Duesberg, W. Steinhögl, M. Liebau, E. Unger, and W. Hönlein: Microelectron. Eng. 64 (2002) 399.

2 J. Li, Q. Ye, A. Cassell, H. T. Ng, R. Stevens, J. Han, and M. Meyyappan: Appl. Phys. Lett. 82 (2003) 2491.

3 T. Wang, K. Jeppson, N. Olofsson, E. E. B. Campbell, and J. Liu: Nanotechnology 20 (2009) 485203.

4 M. Kiuchi, S. Matsui, and Y. Isono: J. Microelectromech. Syst. 16 (2007) 191. 
5 M. Kiuchi, S. Matsui, and Y. Isono: J. Micromech. Microeng. 18 (2008) 065011.

6 H.-J. Oh, H. Omori, M. Sadakata, I. Tsubokura, and Y. Isono: J. Microelectromech. Syst. 23 (2014) 944.

7 S. Wagesreither, E. Bertagnolli, S. Kawase, Y. Isono, and A. Lugstein: Nanotechnology 25 (2014) 455705.

8 M. A. Haque and M. T. A. Saif: Exp. Mech. 42 (2002) 123.

9 D. Zhang, J.-M. Breguet, R. Clavel, V. Sivakov, S. Christiansen, and J. Michler: J. Microelectromech. Syst. 19 (2010) 663.

10 H. D. Espinosa, Y. Zhu, and N. Moldovan: J. Microelectromech. Syst. 16 (2007) 1219.

11 I. Hanasaki, T. Tanaka, Y. Isono, B. Zheng, Y. Uraoka, and I. Yamashita: J. J. Appl. Phys. 50 (2011) 075102.

12 S. D. Senturia: Microsystem Design (Kluwer Academic Publishers Norwell, MA, USA, 2001, ISBN: 0-79237246-8) Chap. 9.

13 D. W. Brenner: Phys. Rev. B 42 (1990) 9458.

14 A. A. Ahmadieh and H. A. Rafizadeh: Phys. Rev. B 7 (1973) 4527.

15 B. Peng, M. Locascio, P. Zapol, S. Li, S. L. Mielke, G. C. Schatz, and H. D. Espinosa: Nature Nanotech. 3 (2008) 626.

16 B. G. Demczyk, Y. M. Wang, J. Cumings, M. Hetman, W. Han, A. Zettl, and R. O. Ritchie: Mater. Sci. Eng. A 334 (2002) 173.

17 L. G. Zhou and S.-Q. Shi: Appl. Phys. Lett. 83 (2003) 1222.

18 P. G. Collins: in Oxford Handbook of Nanoscience and Technology, ed. A. V. Narlikar and Y. Y. Fu (Oxford Univ. Press, USA, 2010, ISBN: 9780199533053) Chap. 2.

19 M.-F. Yu, B. I. Yakobson, and R. S. Ruoff: J. Phys. Chem. B 104 (2000) 8764.

20 A. Kis, K. Jensen, S. Aloni, W. Mickelson, and A. Zettl: Phys. Rev. Lett. 97 (2006) 025501. 